\title{
Deconvolution of ears' activity (DEA): A new experimental paradigm to investigate central auditory processing.
}

\author{
Fabrice Bardy a,b \\ ${ }^{\text {a }}$ HEARing Co-operative Research Centre, Australia \\ ${ }^{\mathrm{b}}$ University of Auckland, New Zealand
}

July 12,2020

\begin{abstract}
A novel experimental paradigm, "deconvolution of ears' activity" (DEA), is presented which allows to disentangle overlapping neural activity from both auditory cortices when two auditory stimuli are presented closely together in time in each ear.

Pairs of multi-tone complexes were presented either binaurally, or sequentially by alternating presentation order in each ear (i.e., first tone complex of the pair presented to one ear and second tone complex to the other ear), using stimulus onset asynchronies (SOAs) shorter than the neural response length. This timing strategy creates overlapping responses, which can be mathematically separated using least-squares deconvolution.

The DEA paradigm allowed the evaluation of the neural representation in the auditory cortex of responses to stimuli presented at syllabic rates (i.e., SOAs between 120 and $260 \mathrm{~ms}$ ). Analysis of the neuromagnetic responses in each cortex offered a sensitive technique to study hemispheric lateralization, ear representation (right versus left), pathway advantage (contra- versus ipsi-lateral) and cortical binaural interaction.

To provide a proof-of-concept of the DEA paradigm, data was recorded from three normalhearing adults. Results showed good test-retest reliability, and indicated that the difference score between hemispheres can potentially be used to assess central auditory processing. This suggests that the method could be a potentially valuable tool for generating an objective "auditory profile" by assessing individual fine-grained auditory processing using a non-invasive recording method.
\end{abstract}




\section{Introduction}

The auditory system is a binaural system. Auditory cortices in right and left hemispheres receive ascending projections originating from each ear. The resulting activity in one cortex is a mixture of signals from both ears. The effects of monaural and binaural stimulation on cortical responses have been studied considerably in humans, using techniques such as magnetoencephalography (MEG) [Pantev et al., 1986]. MEG is well suited to study hemispheric processing differences given the low dispersion of the magnetic field and the location of the cerebral auditory cortical centers in the temporal lobe of each hemisphere. For monaural sound presentation, there is evidence of a predominant contralateral pathway in the human auditory system [Mäkelä et al., 1993; Pantev et al., 1986; Pantev et al., 1998]. The contra-lateral advantage is characterized by shorter latencies and larger amplitudes of the $\mathrm{N} 100 \mathrm{~m}$. These measures reflect anatomical differences, especially the larger number of neurons projecting on the contra-lateral compared to the ipsi-lateral side of the ascending auditory pathways. For binaural presentation at the cortical level, MEG frequency-tagging of cortical steady-state responses can be employed [Fujiki et al., 2002]. Here, stimuli receive a marker, or tag, using a specific modulation frequency. This makes it possible to identify which stimulus evoked the observed cortical response.

The auditory system is a temporally fast system. It can process acoustic stimuli presented with short temporal disparities between the ears. Processing rapidly changing sounds encompasses several levels of transformation from one cochlea to the auditory cortex of both hemispheres. Unfortunately, a noninvasive objective measure of binaural interaction in the auditory cortex during rapid stimulation with temporally restricted sounds is not yet available. However, if such a method were to be available, research on the interaction and/or integration of signals in the auditory cortex for stimuli presented at syllabic rates (i.e., between 4 and $10 \mathrm{~Hz}$ ) could provide new insights into normally developed and disordered central auditory processing systems.

This report describes a novel experimental paradigm, named "deconvolution of ears' activity" (DEA), which makes use of the least-squares (LS) deconvolution technique to allow separation of left and right ear activity in each hemisphere to rapidly presented stimuli [Bardy et al., 2014a; Bardy et al., 2014b]. 
The LS deconvolution technique is a mathematical algorithm designed to disentangle temporally overlapping brain responses. The technique relies on the timing characteristics of the stimulus sequence to be unequally spaced. This specific property is called 'jitter'. In the DEA paradigm, LS deconvolution is applied to a sequence of stimuli presented in pairs either binaurally or sequentially, using stimulus onset asynchronies (SOAs) shorter than the duration of the cortical response. Right and left ear activity is extracted from the mixture of signals in both auditory cortices such that, using this method, the signal propagation from each ear to each auditory cortex can be tracked. The DEA paradigm is introduced in this paper, and is evaluated on three normal hearing adults as a proof-of-concept.

Two hypotheses were investigated: (1) the LS deconvolution technique can disentangle temporally overlapping brain responses in each auditory cortex originating from both ears with a high test-retest reliability; and (2) an auditory profile can be generated based on measures of the auditory pathway lateralization, hemispheric advantage, ear advantage and binaural cortical interaction.

\section{Methods}

Subjects. Test and retest MEG data were obtained from 3 right-handed adult subjects ( 3 males, age: 37, $32,29)$ on two separate occasions. Subjects had no history of neurological or audiological problems and had pure tone audiometric thresholds less than or equal to $20 \mathrm{~dB} \mathrm{HL}$ in all octave frequencies between 250 to $8000 \mathrm{~Hz}$. This study was approved by and conducted under oversight of the Macquarie University Human Research Ethics Committee. All subjects gave written informed consent to participate in this study.

Stimulation. Two multi-tone (MT) stimuli, selected to optimize the amplitude of the cortical response [Bardy et al., 2015], were obtained by amplitude-modulated tone-bursts composed of carrier frequencies of 2 and $1 \mathrm{kHz}$ with modulation frequencies 800 and $400 \mathrm{~Hz}$ respectively. The two MTs were presented in pairs, using jittered SOAs with means of 120, 190 or $260 \mathrm{~ms}$. The jitter distribution, permitting the deconvolution, was rectangular with a width of $70 \mathrm{~ms}$ and a step size of $13.3 \mathrm{~ms}$. The inter-pair interval (IPI), representing the time interval between the onset of two successive pairs of 
stimuli, was jittered with $400 \mathrm{~ms}$ around an average of $1400 \mathrm{~ms}$. The MTs had a rise and decay time of $10 \mathrm{~ms}$, a duration of $50 \mathrm{~ms}$ and an rms intensity of $70 \mathrm{~dB}$ SPL. They were presented through shielded transducers [Oldfield, 1971]. The stimuli were presented in 3 presentation conditions. The first presentation condition was binaural (both stimuli of the pair presented simultaneously to the right and left ears). In the two other presentation conditions, stimuli were alternated sequentially in each ear (i.e., when the left ear received the first tone, the right ear received the second tone of the pair, and vice versa). All 9 conditions ( 3 SOAs x 3 presentation conditions) were randomly presented in a 25 minute long stimulus sequence. In conditions where the cortical response was longer than the SOA, brain responses overlapped in time, and LS deconvolution described by Bardy et al. [2014a] was employed to disentangle the occurring overlapping responses. Thus, for example, in the alternating sequential condition, it was possible within each auditory cortex to separate the activity elicited by the stimulus to the right and left ears respectively from the overlapping cortical response (Figure 1).

Procedure. MEG data were continuously recorded using a whole-head MEG system (Model PQ1160RN2, KIT, Kanazawa, Japan) consisting of 160 coaxial first-order gradiometers with a $50 \mathrm{~mm}$ baseline [Kado et al., 1999; Uehara et al., 2003]. MEG data were acquired in a magnetically shielded room using a sampling rate of $1000 \mathrm{~Hz}$ with a bandpass filter of $0.1-200 \mathrm{~Hz}$ and a $50 \mathrm{~Hz}$ notch filter. For coregistration, the location of five indicator coils placed on the participant's head were digitized. A pen digitizer (Polhemus Fastrack, Colchester, VT) was used to measure the shape of each participant's head which was then carefully centered in the MEG dewar (position error $<10 \mathrm{~mm}$ for each subject). Artefact removal from MEG data included signals exceeding amplitude (>2700 fT/cm) and magnetic gradient ( $>800 \mathrm{fT} / \mathrm{cm} / \mathrm{sample}$ ) criteria [Yetkin et al., 2004]. Averaging and band-pass filtering between $3 \mathrm{~Hz}(6$ $\mathrm{dB} /$ octave, forward) and $30 \mathrm{~Hz}$ (48 dB/octave, zero-phase) was performed for each trigger condition using the non-contaminated epochs. The accepted epochs after artefact rejection were exported from BESA 5.3 into MATLAB (MathWorks, Natick, MA) and downsampled to $100 \mathrm{~Hz}$. Deconvolution was performed for each of the 160 channels to disentangle overlapping responses. For each condition, recovered responses were defined by epochs of $100 \mathrm{~ms}$ pre-stimulus to $380 \mathrm{~ms}$ post-stimulus. 


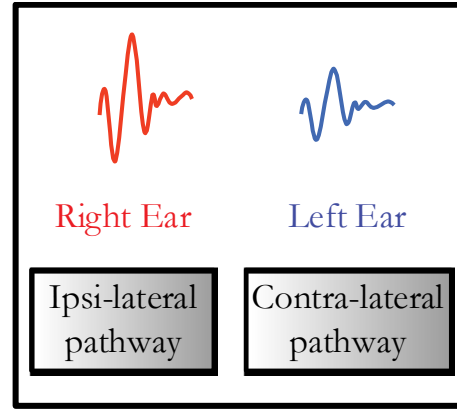

LS Deconvolution

\section{Cortical Responses}

Overlapping response
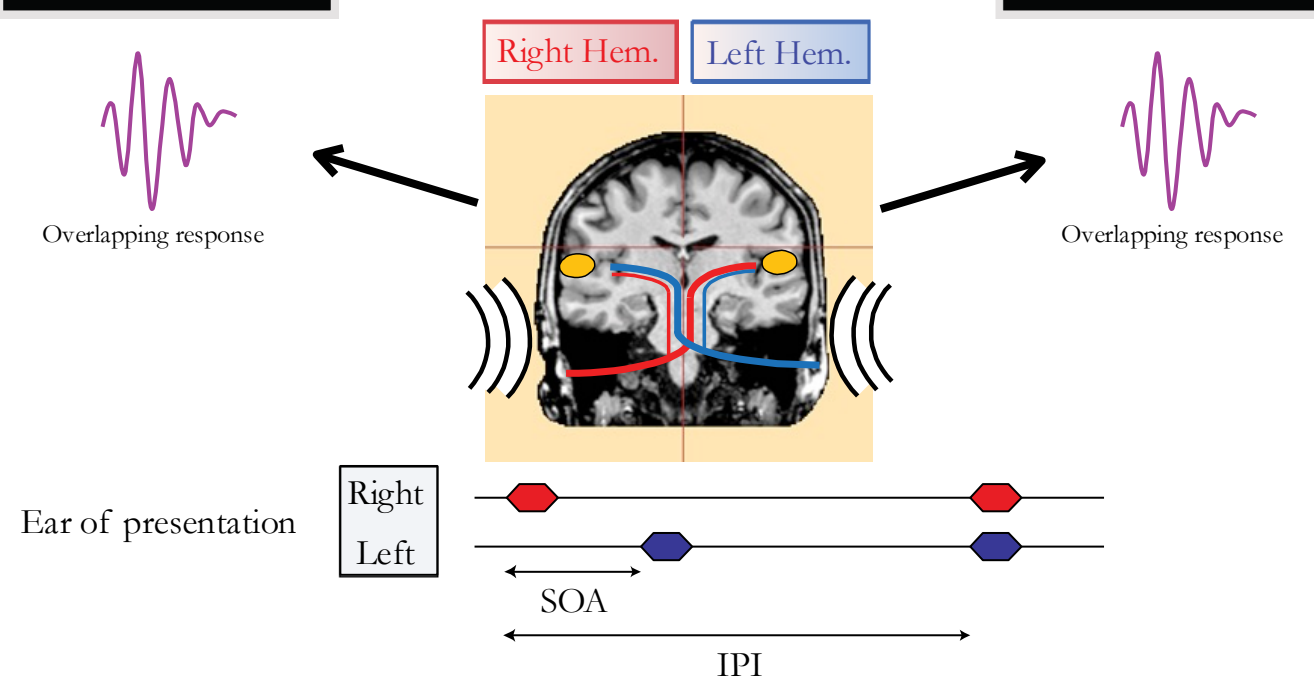

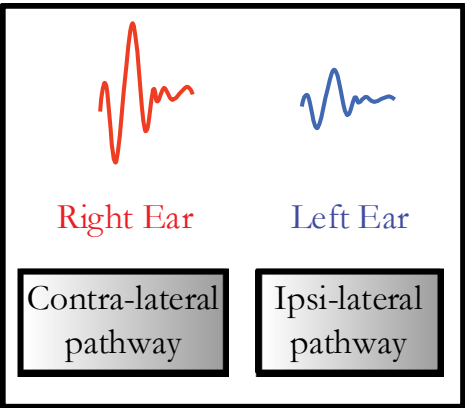

LS Deconvolution

Figure 1. Schematic representation of a sequential condition. The first auditory stimulus of the pair is presented to the right ear, followed closely by a presentation to the left ear. The overlapping neural activity reaches both hemispheres via contra- and ipsi-lateral pathways. The overlapping responses from both ears in each auditory cortex are unwrapped using the leastsquares (LS) deconvolution technique.

Statistical analysis. Amplitudes and latencies were defined by peak measures of magnetic global field power (mGFP) calculated on 40 sensors located over the temporal lobe in each hemisphere. For each subject and each condition, the N100m was defined as the most positive peak in the $80-150 \mathrm{~ms}$ following the sound onset. The selected time window for the P200m was 120-200 ms. Two repeated measures ANOVAs were performed. Greenhouse-Geisser corrections for sphericity were applied, as indicated by the the cited $\varepsilon$ value.

Individual laterality indices (LIs) for hemisphere, pathway, ear and cortical binaural interaction were calculated. For each subject, LIs were calculated based on the relevant mGFP response amplitudes, 
time-averaged over a 200-ms window post-onset. Figure 2 displays an example of auditory cortical responses elicited by pairs of auditory stimuli presented binaurally or alternated sequentially for an individual subject with SOAs jittered around $190 \mathrm{~ms}$. For hemispheric lateralization, the LI was calculated as the difference between left and right mGFP response amplitudes (bottom versus top 6 panels in Figure 2) normalized by the sum of left and right mGFP responses. The LI was +1 for a response geared completely asymmetrical towards the left hemisphere, zero for a symmetrical response, and -1 for a response geared completely asymmetrical towards the right hemisphere. For pathway advantage, the LI was calculated employing the same method using the responses associated with the contra- (panels labeled 3R, 4L, 5L and 6R in Figure 2) and the ipsi-lateral pathways (panels labeled 3L, $4 \mathrm{R}, 5 \mathrm{R}, 6 \mathrm{~L}$ in Figure 2). The ear LI was calculated by comparing mGFP responses from the left ear ( $3^{\text {rd }}$ and $6^{\text {th }}$ columns in Figure 2$)$ to the responses from the right ear $\left(4^{\text {th }}\right.$ and $5^{\text {th }}$ columns in Figure 2$)$. Finally, the binaural interaction LI was computed by comparing binaural stimulation (first 2 columns in Figure 2) and monaural stimulation responses (last 4 columns in Figure 2). The binaural interaction LI was computed for both hemispheres and for each pathway (i.e., ipsi- and contra-lateral). For each subject, the difference between the means for each LI was checked by the Student's t-test. The threshold for significance after Bonferroni correction was $\mathrm{p}<0.0041$. Test-retest reliability indices were obtained using the mean squared error for each measure of LI as well as the intra-class correlation coefficients (ICCs) on mGFP waveforms.

\section{Results}

\section{Cortical responses to rapidly presented stimuli}

Figure 3 presents means and standard deviations of N100m and P200m amplitudes and latencies for ear, stimulus, pathway, and hemisphere. The first ANOVA with hemisphere (right, left), presentation condition (binaural, sequentially alternated left ear first, sequentially alternated right ear first), SOA $(\sim 120, \sim 190, \sim 260 \mathrm{~ms})$, and position (first or second stimulus in the pair) as factors, revealed that the response to the second stimulus of the pair was significantly smaller in amplitude and longer in latency 


\section{SOA $\sim 190(154-226 \mathrm{~ms})$}

Ear of presentation

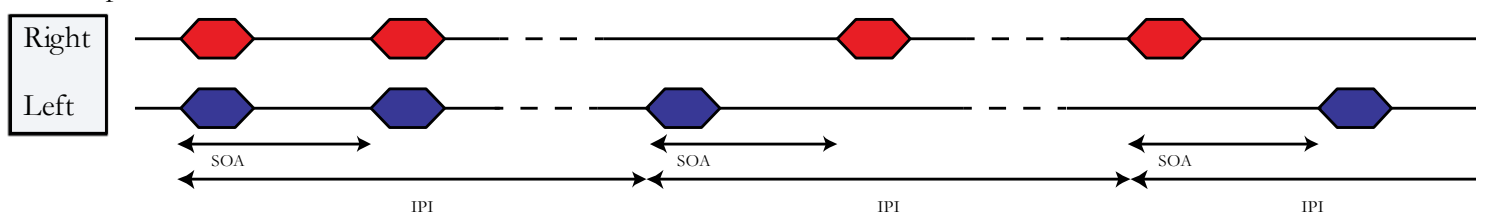

BINAURAL

SEQUENTIALLY ALTERNATED
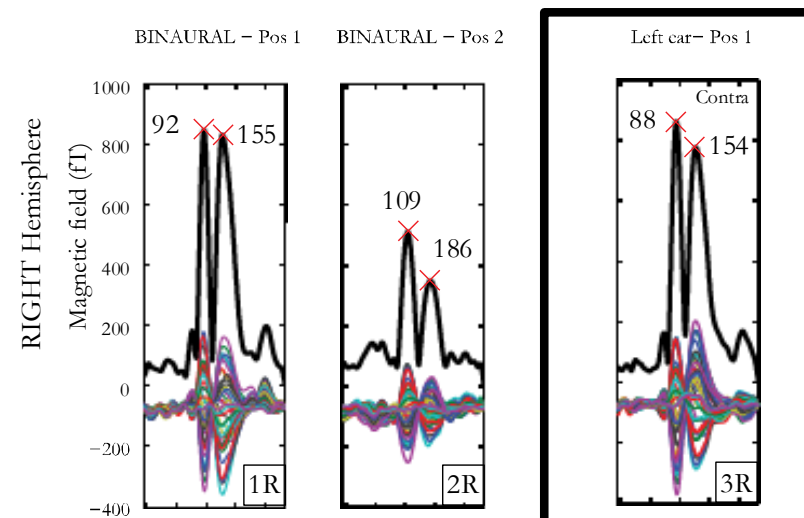

Right car- Pos 2

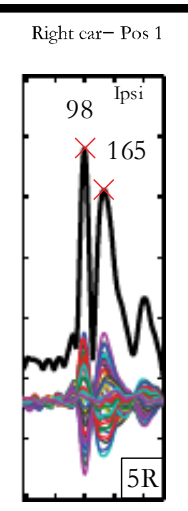

Left ear - Pos 2

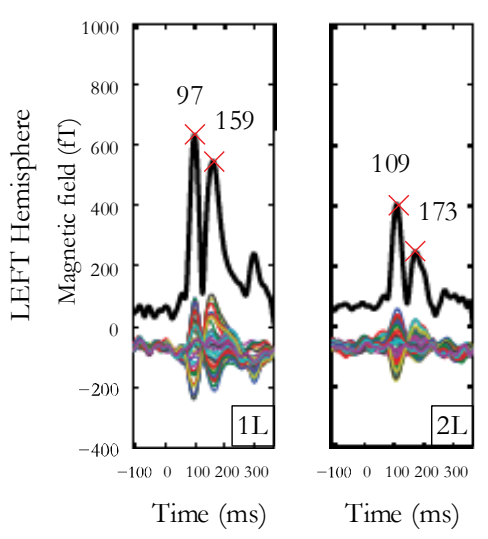

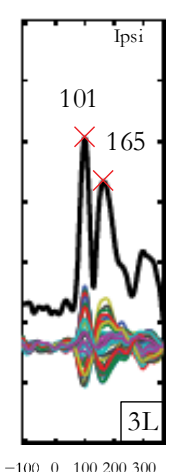

$-100 \quad 0 \quad 100200300$

Time (ms)
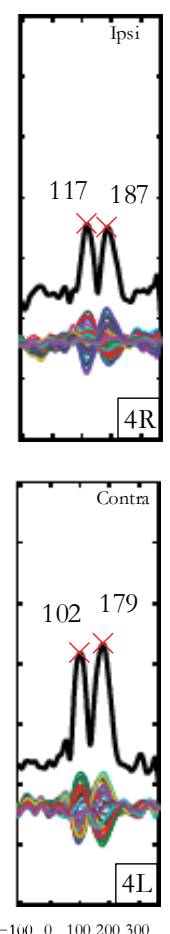

Time (ms)

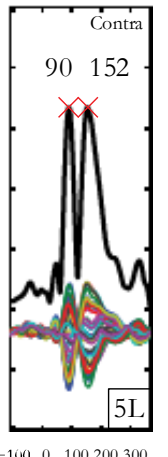

Time (ms)
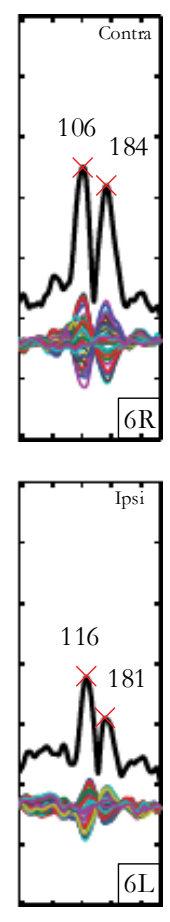

$100 \quad 0100200300$

Time (ms)

Figure 2. Cortical responses from subject 1 for SOAs jittered around $190 \mathrm{~ms}$. Multiple thin waveforms represent activity recorded by each of the 40 sensors located over the temporal lobe, in each hemisphere, after LS deconvolution, from -100 to $380 \mathrm{~ms}$ after stimulus onset. mGFP waveforms are represented with a thick black line, provided for both right and left hemispheres, the 3 presentation conditions ( $1 \mathrm{x}$ binaural, $2 \mathrm{x}$ sequentially alternated) and both first and second tone-bursts. Latencies of the $\mathrm{N} 100 \mathrm{~m}$ and $\mathrm{P} 200 \mathrm{~m}$ are indicated by crosses.

compared to the response to the first stimulus of the pair for both $\mathrm{N} 100 \mathrm{~m}$ (Amp. $\mathrm{F}(1,5)=77.55$, $\mathrm{p}=0.0003, \varepsilon=1 ; \mathrm{F}(1,5)=524.59 ;$ Lat. $\mathrm{p}=0.000003, \varepsilon=1)$ and P200m (Amp. $\mathrm{F}(1,5)=106.95, \mathrm{p}=0.0001$, $\varepsilon=1 ;$ Lat. $\mathrm{F}(1,5)=41.92, \mathrm{p}=0.001, \varepsilon=1)$. 

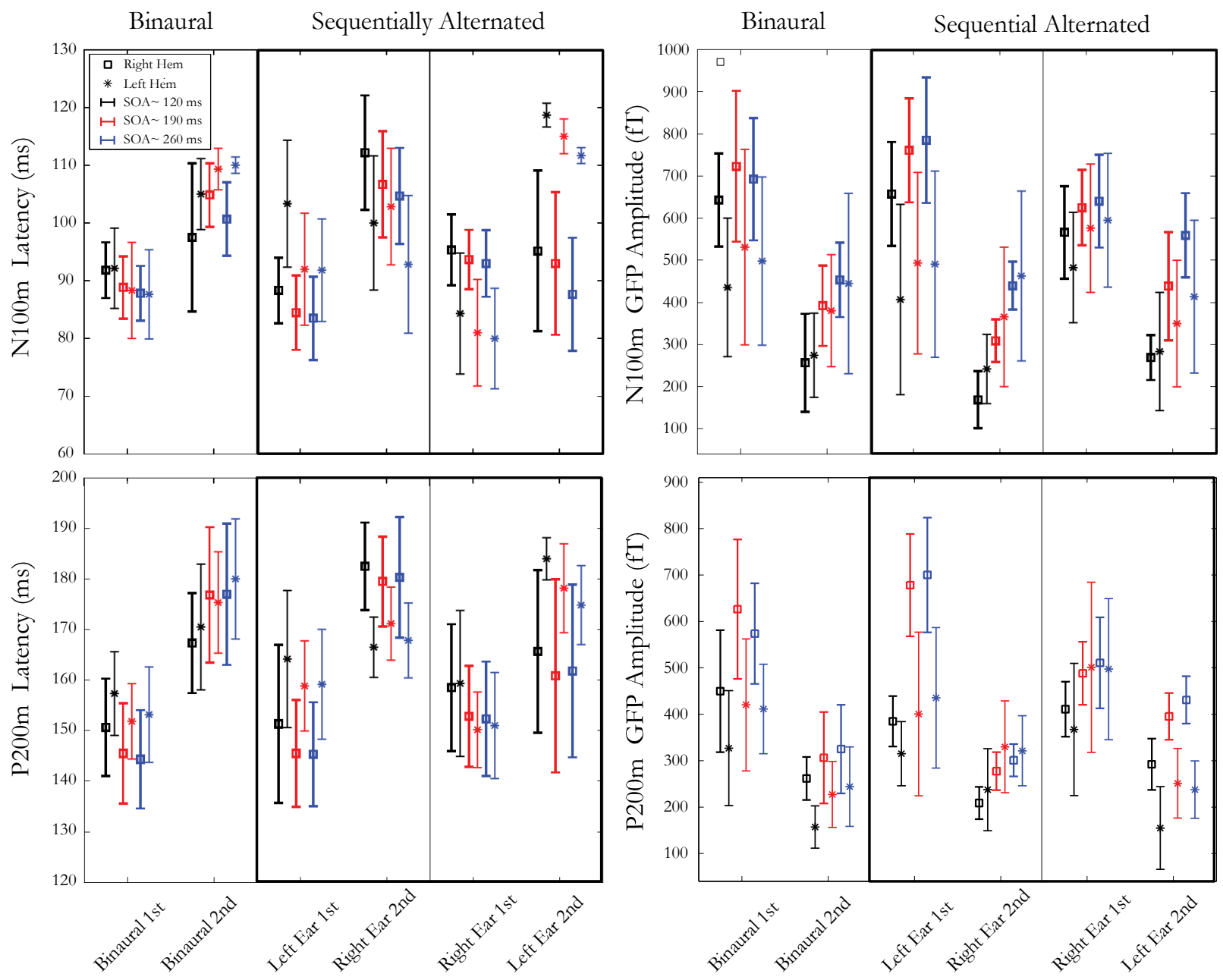

Figure 3. Latencies (left panels) and amplitudes (right panels) of mGFP N100m (top panels) and P200m (bottom panels) components. Each panel represents 3 presentation conditions: 1 binaural condition and 2 sequentially alternated conditions (stimulus presented first at either left or right ear). Within each presentation condition, three SOAs $(\sim 120, \sim 190$ and $\sim 260 \mathrm{~ms})$ are used, resulting in two responses to both stimuli of the pair, recorded from both right and left hemispheres. Error bars denote standard deviations between participants.

The second ANOVA similarly used hemisphere, presentation condition and SOA as factors, but only considered responses to the second stimulus in the pair. The effect of SOA was found to be significant for both amplitudes and latencies of N100m (Amp. $\mathrm{F}(2,10)=46.48, \mathrm{p}=0.000009, \varepsilon=0.58$; Lat. $\mathrm{F}(2,10)=7.30, \mathrm{p}=0.03, \varepsilon=0.54)$ and for $\mathrm{P} 200 \mathrm{~m}$ amplitude $(\mathrm{F}(2,10)=53.95, \mathrm{p}=0.000004, \varepsilon=0.78)$. The cortical responses to the second stimulus in the pair increased in amplitude and decreased in latency for longer SOAs when compared to shorter SOAs. Moreover a significant interaction was present between 
SOA and presentation condition for both $\mathrm{N} 100 \mathrm{~m}(\mathrm{~F}(4,20)=10.07 ; \mathrm{p}=0.001, \varepsilon=0.60)$ and $\mathrm{P} 200 \mathrm{~m}$ $(\mathrm{F}(4,20)=8.29 ; \mathrm{p}=0.004, \varepsilon=0.60)$ latencies. A decrease in response latency was observed when SOA increased in the sequentially alternated presentation condition, while this trend was inverted in the binaural presentation conditions.

\section{Hemispheric lateralization}

The hemispheric lateralization index (LI) for response amplitude presented in Figure 4a shows intraindividual differences on the vertical abscissa, and inter-individual differences on the horizontal abscissa. Subject 1 presented a rightward, subject 2 a large rightward, and subject 3 a slightly leftward lateralization. The t-test, which allows comparing the hemispheric LI to 0 , was significant for each subject $(\mathrm{p}<0.001)$ after Bonferroni correction. No differences in symmetrical activation were found for the latencies either for subject $1(\mathrm{p}=0.86)$ or subject $2(\mathrm{p}=0.51)$. However, significantly earlier latencies were found in the right hemisphere for subject $3(\mathrm{p}=0.0003)$.

\section{Pathway advantage}

The pathway LI calculated by contrasting contra- versus ipsi-lateral pathway responses in the sequential conditions is represented in Figure $4 \mathrm{~b}$. After Bonferroni correction, significantly larger amplitudes and shorter latencies for the N100m and P200m were measured in the contra-lateral pathway for all subjects $(\mathrm{p}<0.0001)$.

\section{Ear advantage}

The statistical results of ear LI presented in Figure $4 \mathrm{c}$ indicated no significant amplitude difference between the activity elicited by the right and the left ear for subject $1(\mathrm{p}=0.96)$ and for subject 2 $(\mathrm{p}=0.01)$. A left ear advantage was observed for subject 3 for both amplitude $(\mathrm{p}=0.002)$ and latency $(\mathrm{p}=0.002)$. 


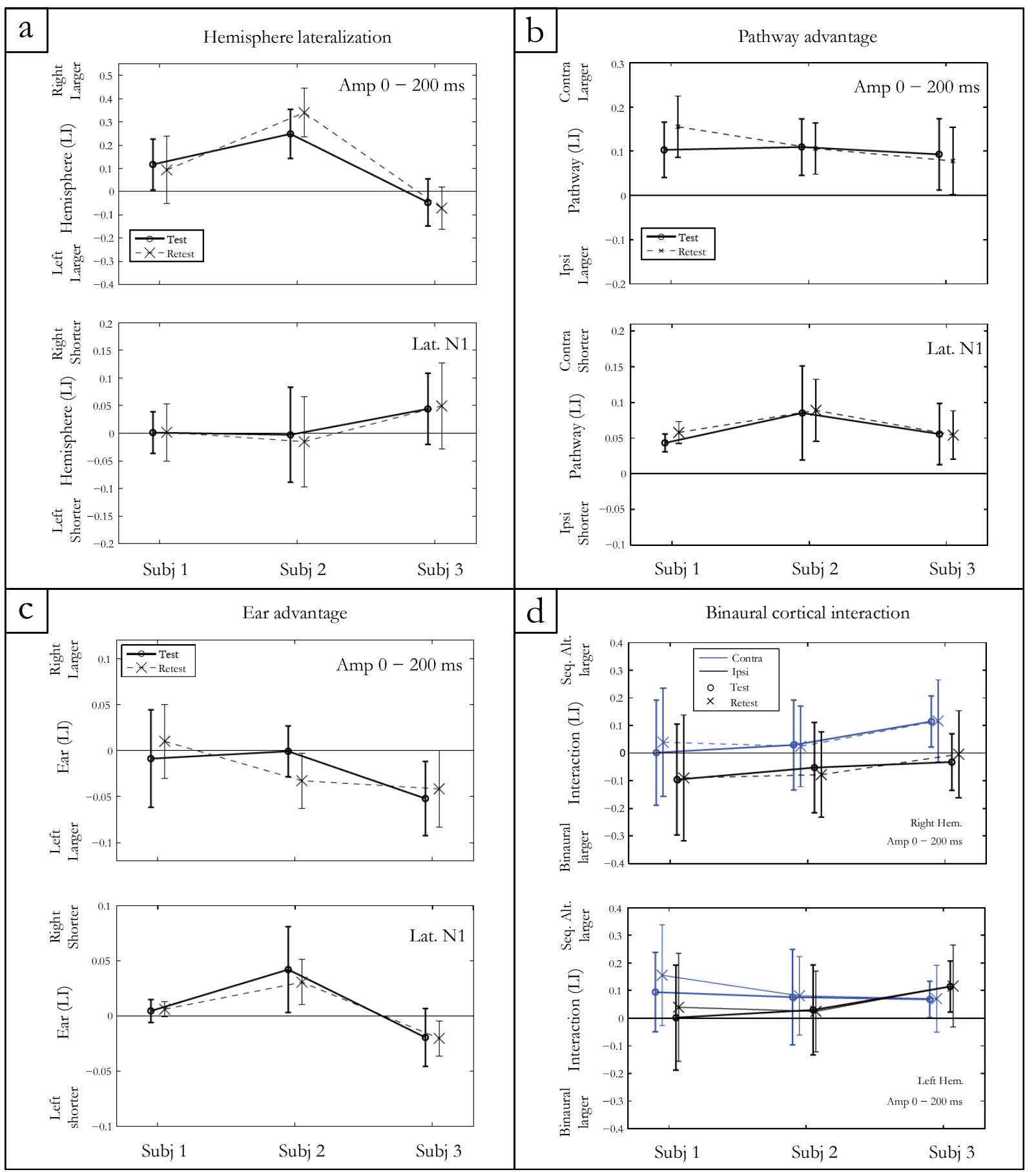

Figure 4. Indices of hemispheric lateralization, pathway advantage, and ear advantage for mGFP amplitudes in a $200 \mathrm{~ms}$ post onset window, and for $\mathrm{N} 100 \mathrm{~m}$ latency. Both test and retest conditions are shown. The binaural cortical interaction is represented for the mean mGFP amplitude in the right and left hemisphere for the contra- and ipsi-lateral pathways. Error bars denote standard deviations between conditions for each participant. 


\section{Cortical binaural interaction (CBI)}

Figure $4 \mathrm{~d}$ shows the CBI for the 3 subjects in both hemispheres for contra- and ipsi-lateral pathways. The finding of a positive CBI LI indicates that the response recorded in the sequentially alternated condition is larger compared to the response in the ipsi-lateral pathway. CBI of different natures are observed for each subject. When collapsed across hemispheres, the t-test showed that CBI was close to significance only for subject 3 (subject $1: \mathrm{p}=0.02$; subject $2: \mathrm{p}=0.10$; subject 3 : $\mathrm{p}=0.006$ ).

\section{Test-retest reliability}

Two different test-retest reliability measures were computed. First, the mGFP waveforms were compared for test and retest conditions by computing the intra-class correlation coefficients (ICCs) for the 3 subjects in a $250 \mathrm{~ms}$ window post onset. A mean ICC value larger than 0.75 for each subject (i.e., subject $1=0.78$, subject $2=0.79$; subject $3=0.84$ ) demonstrated a good test-retest reliability.

Second, a test-retest index was calculated using the mean squared error (mean $=0.057 ; \mathrm{SD}=0.026)$ of all four indices presented in Figure 4 (i.e. hemispheric lateralization, pathway advantage, ear advantage and $\mathrm{CBI})$.

\section{Discussion}

The central aim of this paper was to introduce the deconvolution of ears' activity (DEA) paradigm which disentangles the activity in both auditory cortices elicited by stimuli presented to both ears simultaneously or separately. In this paradigm, the LS deconvolution technique was applied to MEG data recorded using pairs of stimuli presented either binaurally or alternating sequentially (i.e. right-left and left-right). The DEA paradigm allowed the investigation of auditory information transfer from one specific ear to both auditory cortices. It could also be used to explore response lateralization, the strength of crossed auditory pathways and the response adaptation properties to auditory stimuli closely separated in time. Furthermore, it allowed for the investigation of non-linear processing in the brain and 
CBI, mainly caused by inhibition mechanisms [Imig and Brugge, 1978; Imig and Reale, 1981; Papanicolaou et al., 1990; Reite et al., 1981].

We demonstrated the feasibility and test - retest reproducibility of this non-invasive measure on 3 righthanded normal-hearing subjects. The case studies provided examples of different auditory processing characteristics at the cortical level, identifiable at the individual level. The inter-individual differences were detectable by assessment of the difference in response between experimental conditions. For example, hemispheric lateralization was assessed by computation of the LI calculated from the responses in each hemisphere. The CBI was investigated by contrasting binaural and monaural stimulation both in contra- and ipsi-lateral pathways. The results collected using the DEA paradigm allows an objective auditory processing characterization and the generation of an individual "auditory profile" in a relatively quick time (i.e., $25 \mathrm{~min}$ ).

\section{Experimental results:}

The data recorded from 3 normal-hearing subjects confirmed that both ears were represented in each cortical hemisphere. However, differences in latency and amplitude were observed for each response to various conditions.

Beyond the idea proposed by Poeppel [2003] that sound processing in the brain is a bilateral phenomenon, the present study revealed inter-individual differences in the hemispheric lateralization of the cortical response. While two subjects showed a rightward hemisphere lateralization for response amplitude, the third subject had a leftward lateralization. These hemispheric asymmetries and specializations for processing auditory stimuli were also reported previously by Mäkelä et al. [1993] and Jamison et al. [2006]. The cerebral lateralization of the auditory cortical area however is still highly debated [Bishop, 2013; Scott and McGettigan, 2013].

For all subjects tested, the N100m was larger and approximately $10 \mathrm{~ms}$ shorter for the contra-lateral compared to the ipsi-lateral auditory pathway in the sequentially alternated conditions. These results 
are in agreement with several studies showing a contra-lateral dominance based on lateralization of the N100m component [Pantev et al., 1986; Pantev et al., 1998; Tiihonen et al., 1989; Woldorff et al., 1999].

Individual differences were also observed when comparing ear activity. Further research will need to investigate whether this objective measure of ear advantage is correlated with behavioral performance on a dichotic listening task such as the Dichotic Digits Test [Musiek, 1983].

The DEA paradigm allowed to investigate the suppression-type interaction and neural mechanisms underlying the processing of rapidly presented signals. As shown in Figure $4 \mathrm{~d}$, different binaural interactions were observed. Amplitudes of responses elicited in the sequentially alternated presentation condition were found to be either slightly larger, slightly smaller or of similar amplitude compared to the binaural presentation condition. Inter-subject differences were observed with different interactions depending on hemisphere and pathway involved. A MEG study using complex tones showed that responses to ipsi-lateral stimuli over the right auditory cortex are inhibited by the stimuli presented in the contra-lateral (left) ear [Brancucci et al., 2004].

Lastly, cortical responses to stimulus pairs separated by short SOAs allowed the study of the representation in the auditory cortex of stimuli presented closely together. Results presented in Figure 3 showed a large decrease in amplitude and increase in latency of the cortical response when it stimulus was closely preceded by another auditory stimulus.

We conclude that the DEA paradigm could represent a technique to study interesting properties of the central auditory system. Individual differences are of special interest as they provide an alternative characterization of the hearing profile of a person which could potentially be useful to for example objectively identify auditory processing disorder (APD) subjects. Using the LS deconvolution technique to separate overlapping ear activity in both auditory cortices, recorded MEG data can provide a measure for rapid temporal processing, response lateralization, auditory pathway and ear advantage, and CBI for rapidly presented sound stimuli. Such a test would allow studying the temporal acuity of the human auditory system when processing rapid changes in the acoustic signal. Moreover, it could provide insights concerning the flow of neural signals from the cochlea to the cerebral cortex. From a clinical 
perspective, tests are needed to better evaluate and understand the neurological characteristics of binaural processing occurring in the auditory system. Such tests could contribute to the diagnosis of APDs or neurodevelopment disorders, such as specific language impairment (SLI) or dyslexia where abnormal crossing pathways or the disability to process rapid auditory stimuli has been identified [Lamminmäki et al., 2012]. More complex sounds, such as speech syllables (using carefully selected jitter parameters), could be used in the future to investigate the influence of stimuli on binaural interaction mechanisms and lateralization of the response.

\section{Acknowledgments}

This work was supported in part by: the HEARing CRC, established and supported under the Australian Cooperative Research Centres Program, an Australian Government Initiative, by the Australian Government Department of Health and by the Oticon Foundation. The authors gratefully thank Bram Van Dun, Harvey Dillon, Catherine McMahon, Robert Cowan and Ramesh Rajan for their suggestions during the preparation of this manuscript. 


\section{References}

Bardy F, Dillon H, Van Dun B: Least-squares deconvolution of evoked potentials and sequence optimization for multiple stimuli under low-jitter conditions. Clin Neurophysiol 2014a; $125: 727-737$.

Bardy F, Van Dun B, Dillon H: "Bigger is better: Increasing cortical response amplitude via stimulus spectral complexity". Ear Hear, 2015, 36: 677-687.

Bardy F, Van Dun B, Dillon H, McMahon CM: Deconvolution of overlapping cortical auditory evoked potentials (caeps) recorded using short stimulus onset-asynchrony (soa) ranges. Clin Neurophysiol 2014b;125:814-826.

Bishop DV: Cerebral asymmetry and language development: Cause, correlate, or consequence? Science 2013;340.

Brancucci A, Babiloni C, Babiloni F, Galderisi S, Mucci A, Tecchio F, Zappasodi F, Pizzella V, Romani GL, Rossini PM: Inhibition of auditory cortical responses to ipsilateral stimuli during dichotic listening: Evidence from magnetoencephalography. Eur J Neurosci 2004;19:23292336.

Fujiki N, Jousmaki V, Hari R: Neuromagnetic responses to frequency-tagged sounds: A new method to follow inputs from each ear to the human auditory cortex during binaural hearing. $\mathrm{J}$ Neurosci 2002;22:RC205 (201-204).

Imig TJ, Brugge JF: Sources and terminations of callosal axons related to binaural and frequency maps in primary auditory cortex of the cat. J Comp Neurol 1978;182:637-660.

Imig TJ, Reale RA: Ipsilateral corticocortical projections related to binaural columns in cat primary auditory cortex. J Comp Neurol 1981;203:1-14.

Jamison HL, Watkins KE, Bishop DV, Matthews PM: Hemispheric specialization for processing auditory nonspeech stimuli. Cereb Cortex 2006;16:1266-1275.

Kado H, Higuchi M, Shimogawara M, Haruta Y, Adachi Y, Kawai J, Ogata H, Uehara G: Magnetoencephalogram systems developed at kit. IEEE Trans Appl Supercond 1999;9:40574062. 
Lamminmäki S, Massinen S, Nopola-Hemmi J, Kere J, Hari R: Human robo1 regulates interaural interaction in auditory pathways. J Neurosci 2012;32:966-971.

Mäkelä JP, Ahonen A, Hämäläinen M, Hari R, Llmoniemi R, Kajola M, Knuutila J, Lounasmaa O, McEvoy L, Salmelin R: Functional differences between auditory cortices of the two hemispheres revealed by whole-head neuromagnetic recordings. Hum Brain Mapp 1993;1:4856.

Musiek FE: Assessment of central auditory dysfunction: The dichotic digit test revisited. Ear Hear $1983 ; 4: 79-83$.

Oldfield RC: The assessment and analysis of handedness: The edinburgh inventory. Neuropsychologia 1971;9:97-113.

Pantev C, Lütkenhöner B, Hoke M, Lehnertz K: Comparison between simultaneously recorded auditory-evoked magnetic fields and potentials elicited by ipsilateral, contralateral and binaural tone burst stimulation. Int J Audiol 1986;25:54-61.

Pantev C, Ross B, Berg P, Elbert T, Rockstroh B: Study of the human auditory cortices using a whole-head magnetometer: Left vs. Right hemisphere and ipsilateral vs. Contralateral stimulation. Audiol Neurootol 1998;3:183-190.

Papanicolaou AC, Baumann S, Rogers RL, Saydjari C, Amparo EG, Eisenberg HM: Localization of auditory response sources using magnetoencephalography and magnetic resonance imaging. Arch Neurol 1990;47:33-37.

Poeppel D: The analysis of speech in different temporal integration windows: Cerebral lateralization as 'asymmetric sampling in time'. Speech Commun 2003;41:245-255.

Reite M, Zimmerman JT, Zimmerman JE: Magnetic auditory evoked fields: Interhemispheric asymmetry. Electroencephalogr Clin Neurophysiol 1981;51:388-392.

Scott SK, McGettigan C: Do temporal processes underlie left hemisphere dominance in speech perception? Brain Lang 2013;127:36-45.

Tiihonen J, Hari R, Kaukoranta E, Kajola M: Interaural interaction in the human auditory cortex. Int J Audiol 1989;28:37-48. 
Uehara G, Adachi Y, Kawai J, Shimogawara M, Higuchi M, Haruta Y, Ogata H, Hisashi K: Multichannel squid systems for biomagnetic measurement. IEICE trans electron 2003;86:43-54.

Woldorff MG, Tempelmann C, Fell J, Tegeler C, Gaschler-Markefski B, Hinrichs H, Heinze H-J, Scheich H: Lateralized auditory spatial perception and the contralaterality of cortical processing as studied with functional magnetic resonance imaging and magnetoencephalography. Hum Brain Mapp 1999;7:49-66.

Yetkin FZ, Roland PS, Christensen WF, Purdy PD: Silent functional magnetic resonance imaging (fmri) of tonotopicity and stimulus intensity coding in human primary auditory cortex. Laryngoscope 2004;114:512-518. 